\title{
A Low-Overhead Cooperative Retransmission Scheme for IR-UWB Networks
}

\author{
Ghasem Naddafzadeh Shirazi, ${ }^{1,2}$ Peng-Yong Kong, ${ }^{1,2}$ and Chen-Khong Tham ${ }^{1,2}$ \\ ${ }^{1}$ Department of Electrical \& Computer Engineering, Faculty of Engineering, National University of Singapore, Singapore 117576 \\ ${ }^{2}$ Institute for Infocomm Research, Agency for Science, Technology, and Research (A*STAR), Singapore 138632
}

Correspondence should be addressed to Ghasem Naddafzadeh Shirazi, naddafzadeh@nus.edu.sg

Received 29 September 2008; Accepted 3 December 2008

Recommended by Luca De Nardis

The UWB unique properties such as fine ranging and immunity to small scale fading are utilized in order to exploit the multiuser diversity in UWB networks. The optimal cooperation strategies in the absence of control packet overhead are analyzed in the proactive and reactive settings. It is shown that the proposed method achieves a considerable diversity gain while minimizing the overhead of control packet exchange that is required for coordination among the relays.

Copyright (C) 2008 Ghasem Naddafzadeh Shirazi et al. This is an open access article distributed under the Creative Commons Attribution License, which permits unrestricted use, distribution, and reproduction in any medium, provided the original work is properly cited.

\section{Introduction}

Due to the large bandwidth occupied by pulses, UWB signals are considered robust to small scale fading effects. In addition, UWB enables high accuracy ranging which can be used for the design of location-aware MAC and routing mechanisms. We exploit these properties of UWB, that is, availability of ranging information and immunity to small scale fading, in the design of an UWB-based Cooperative Retransmission Scheme (UCoRS) [1].

Most of the existing distributed relay selection schemes, such as [2-4] rely on the Priority-Based Backoff Timer $(P B T)$ mechanism to discover which relay is the best one at a time instance by sending a flag message. We note that like $P B T$, the other existing mechanisms such as CMAC [5] also require the exchange of the RTS/CTS and other control messages for every transmission. These cooperative methods may be inefficient for UWB networks. This is because the standard IR-UWB MAC protocol is ALOHA [6] and the exchange of RTS/CTS packets is not required prior to the data transmission in UWB. Furthermore, it is preferred to exchange fewer control packets due to the complex and costly UWB receiving procedure.

\section{System Model}

Figure 1 shows the system model. As can be seen, there are a source $S$ and a destination $D$, and $N$ relays $R_{i}, i=$
$1,2, \ldots, N$, in a slotted time domain, and each time slot consists of 2 subslots. At the transmission subslot (Tx), the source node sends data to its destination. At the Cx subslot, the relays retransmit the source data. In particular, the $i$ th relay, $R_{i}$, decides to cooperate (i.e., retransmit the data) with probability $a_{i}$.

Since accurate ranging information is available through UWB physical layer, we also presume that when $R_{i}$ finds its distance to $S$ and $D$, it broadcasts a packet to inform other nodes about these ranging information. Note that as long as the nodes do not move, the process of ranging and informing other nodes should be performed only once, which incurs much less overhead compared to sending control packets for every transmission.

The link success probabilities are denoted by $P_{i}$ and $Q_{i}$, as can be seen in Figure 1. The success probability of the $S-D$ link is denoted by $P_{0}$. To calculate these values, we note that in time-hopping pulse position modulation, THPPM, the transmitted signal by node $i$ is given by $s^{i}(t)=$ $\sum_{j=-\infty}^{\infty} \sqrt{E_{p}} \omega\left(t-j T_{f}-c_{j}^{i} T_{c}-\delta b_{\left\lfloor j / N_{s}\right\rfloor}^{i}\right)$, where $E_{p}$ is the transmission energy per pulse, $T_{f}$ and $T_{c}$ are the frame and chip durations, $b_{\left\lfloor j / N_{s}\right\rfloor}^{i} \in\{0,1\}$ is the information bit to be sent, $\omega(t)$ is the monocycle pulse, and $\delta$ determines the time shift in the chip when the data bit is 1 . Each frame consists of $N_{h}$ chips, that is, $T_{f}=N_{h} T_{c}$. Moreover, each bit is repeated in $N_{S}$ frames with different time hopping 
codes, $c_{j}^{i} \in\left\{0,1, \ldots, N_{h}-1\right\}$, which results in additional (random) time shifts and hence increases the pulse immunity to interference.

The received signal from user $i$ at node $j$ is given by [7] $r^{i j}(t)=\alpha^{i j} \sum_{c=1}^{C} \sum_{l=1}^{L} \beta_{c l s} s^{i}\left(t-\tau_{c l}\right)+n(t)$, where $n(t)$ is AWGN with the power spectral density $N_{0} / 2$, and $\alpha^{i j}$ denotes the $i-j$ link gain. Since UWB pulses are robust to small scale fading effects, we consider only the channel pathloss, as defined in $[1,6]$. Then, the bit error probability (BEP) in the absence of interference can be approximated by $P_{\text {be }}\left(d_{i j}\right)=(1 / 2) \operatorname{erfc}\left(\sqrt{\left(\alpha^{i j} E_{p} N_{S} / 2 N_{0}\right)(1-\rho(\delta))}\right)$ [7], where $\rho(\delta)$ is the autocorrelation function of the monocycle pulse, $\omega(t)$. From the above-mentioned model, the probability that a packet with length $L$ bits is successfully transmitted can be represented as follows:

$$
P_{s}(d)=1-\left(1-P_{\mathrm{be}}(d)\right)^{L} .
$$

This equation can be used to determine the values of $P_{i}$ and $Q_{i}$ as a function of the relays' distances to $S$ and $D$. Having obtained $P_{i}$ and $Q_{i}$ s from (1), the next problem is to find the cooperation probabilities $a_{i}$ in order to maximize the $S-D$ throughput.

\section{Analysis}

We assume that the packet level collision occurs if the signal strength of more than one packet is above the threshold at the receiver. Therefore, $D$ successfully receives a useful data packet if either the $S-D$ transmission in the Tx subslot is successful, or the transmission from one and only one of the relays in the Cx subslot is successful.

We consider two different settings, namely, the proactive and reactive modes. In the proactive mode, the decision is made prior to the source transmission. In the reactive mode, all relays listen for the data first and then decide to cooperate. Note that since message exchange between relays is not performed in $U C o R S$, a relay $R_{i}$ is unable to find out the set of relays which have successfully decoded the packet from $S$ at time slot $t$, denoted by $F(t)$. In fact, the global optimum of the relay selection problem would be obtained if $F(t)$ were available to the nodes.

In the proactive case, the expected success probability in a time slot is given by

$$
U(A)=P_{0}+\left(1-P_{0}\right) \sum_{i=1}^{N}\left[a_{i} P_{i} Q_{i} \prod_{j=1, j \neq i}^{N}\left(1-a_{j} P_{j} Q_{j}\right)\right] .
$$

In order to find the optimal solution of (2), we use Lemma 1 in the Appendix. The following theorem gives the optimal solution.

Theorem 1. Consider a cooperative network with one S-D pair and $N$ relays. The optimal cooperation strategy to maximize the $S$-D throughput $\left(U(A)\right.$ in (2)) is $A^{(K)}=\left\{a_{i}=1, i \leq\right.$ $\left.K ; a_{i}=0, i>K\right\}$, where $K$ satisfies: $\sum_{i=1}^{K-1}\left(P_{i} Q_{i} /\left(1-P_{i} Q_{i}\right)\right)<$ 1 , and $\sum_{i=1}^{K}\left(P_{i} Q_{i} /\left(1-P_{i} Q_{i}\right)\right) \geq 1$, where relays are sorted in descending order according to the values of $P_{i} Q_{i}$ (i.e., $i \leq j \Leftrightarrow$

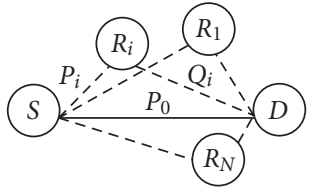

(a) Network model

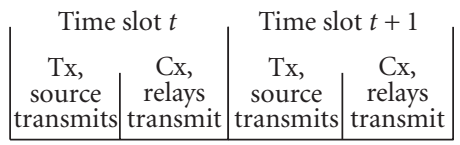

(b) Time slot model
Figure 1: System model.

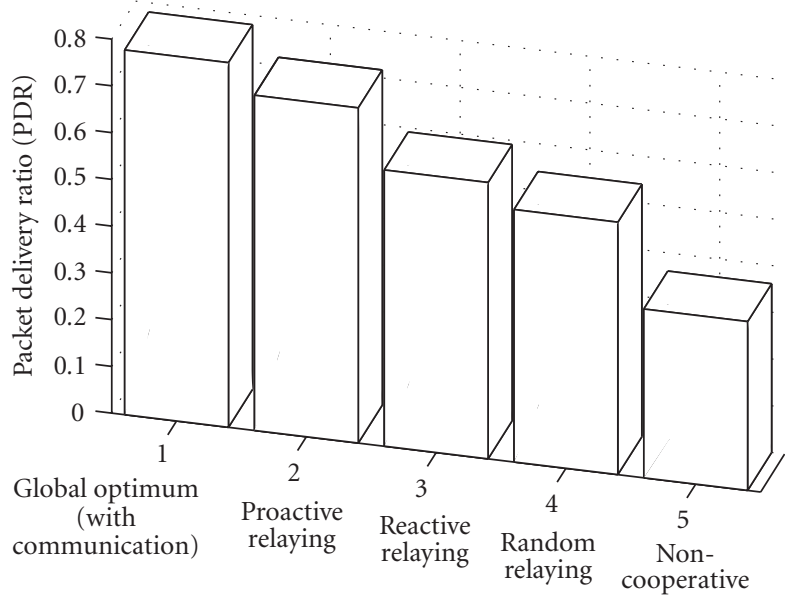

FIGURE 2: Comparison of packet delivery ratio (PDR) for different schemes.

$\left.P_{i} Q_{i} \geq P_{j} Q_{j}\right)$; and $A^{(K)}$ denotes a binary vector whose first Kth elements are 1. (Proof is straightforward from Lemma 1.)

Here, we mention that if $P_{1} Q_{1} \geq 0.5$, then $K=1$, and only $R_{1}$ will be active. In this special case, the result is in agreement with [3]. The reactive and global optimum cooperation strategies can be derived using the same reasoning as Theorem 1, as discussed in detail in [1].

\section{Performance Evaluation}

Figure 2 compares the packet delivery ratio (PDR) for different scenarios. As can be seen, the proactive performance is near to the maximum achievable throughput. Furthermore, as expected, both reactive and proactive methods outperform the noncooperative case.

Figure 3(a) shows the effect of increasing the number of relays on the achieved PDR in UCoRS for different $S$ - $D$ link qualities. As can be seen, adding one relay can significantly increase the PDR of the direct link. However, the achieved PDR in UCoRS is upper bounded by a function of $d_{S D}$, regardless of number of available relays.

Figure 3(b) shows the asymptotic achievable throughput of $U C o R S, P B T$, and noncooperative schemes as a function of $P_{0}$ when $N \rightarrow \infty$. As stated previously, the throughput advantage of PBT over UCoRS is at the expense of control packet exchange for every data transmission, which may not be efficient in UWB. More details can be found in [1]. 


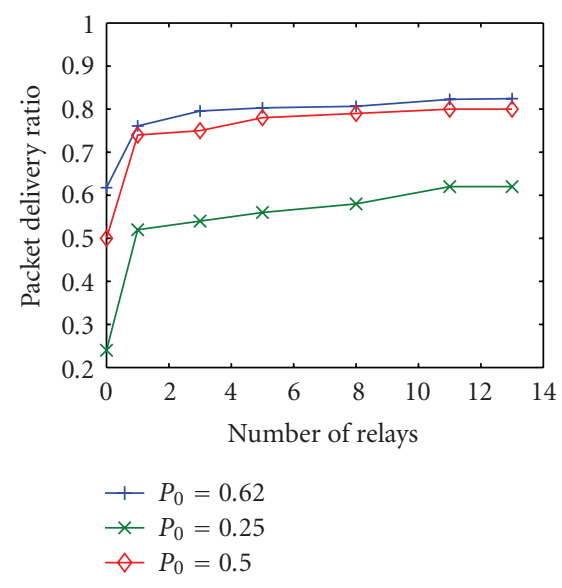

(a) Effect of increasing number of relays on PDR

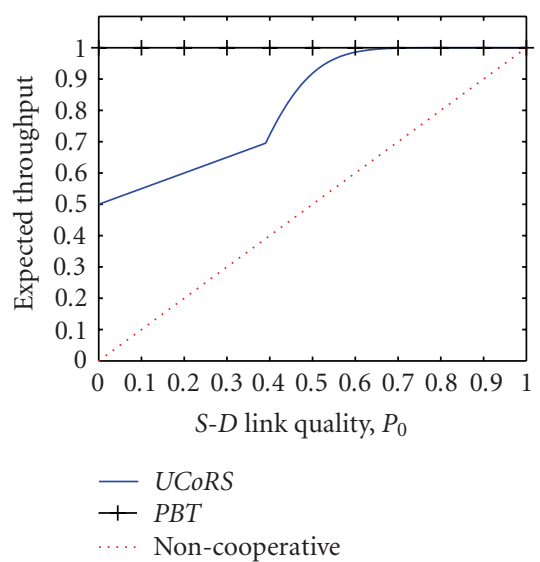

(b) Asymptotic throughput achievable by $U C O R S$ and $P B T$ as $N \rightarrow \infty$

FIGURE 3: Simulation results.

\section{Conclusion}

We introduced UCoRS, a simple UWB-based Cooperative Retransmission Scheme, that utilizes the unique properties of IR-UWB technology for achieving multiuser diversity in UWB in the proactive and reactive settings. The amount of control packet overhead is minimized in UCoRS in order to eliminate the corresponding energy cost at the UWB receivers.

\section{Appendix}

Lemma 1. Assume a set of variables $Z=\left\{z_{i}\right\}, i=1,2, \ldots, n$, that can take on real values between 0 and $1>m_{1} \geq m_{2} \geq$ $\cdots \geq m_{n}$, respectively. Then, the maximum value of $X(Z)=$ $\sum_{i=1}^{n}\left(z_{i}\right) \prod_{j \neq i}\left(1-z_{j}\right)$ is obtained when $z_{i}=m_{i}, i \leq K$, and $z_{i}=0, i>K$, where $K$ satisfies $\sum_{i=1}^{K}\left(m_{i} /\left(1-m_{i}\right)\right) \geq 1$, and $\sum_{i=1}^{K-1}\left(m_{i} /\left(1-m_{i}\right)\right)<1$.

Proof. Taking the partial derivative of $X(Z)$, we have

$$
\begin{aligned}
\frac{\partial X(Z)}{\partial z_{i}} & =\prod_{j \neq i}\left(1-z_{j}\right)-\sum_{j \neq i}\left(z_{j} \prod_{k \neq i, j}\left(1-z_{k}\right)\right) \\
& =\prod_{j \neq i}\left(1-z_{j}\right)\left(1-\sum_{j \neq i} \frac{z_{j}}{1-z_{j}}\right) .
\end{aligned}
$$

Therefore, $\partial X(Z) / \partial z_{i}>0 \Leftrightarrow \sum_{j=1, j \neq i}^{n}\left(z_{j} /\left(1-z_{j}\right)\right)<$ 1 , and $\partial X(Z) / \partial z_{i}>\partial X(Z) / \partial z_{j} \Leftrightarrow z_{i}>z_{j}$. According to these two results, in order to maximize $X(Z)$, the $K$ "best" variables (with looser bounds) should be set to their maximum values and other variables should be set to 0 . The required conditions on $K$ are also clearly observed from the above-mentioned equations. Note that if $m_{1} \geq 0.5$, then $K=1$.

\section{Acknowledgments}

An earlier version of paper [1] has won the Best Student Paper award in the IEEE International Conference on UltraWideband (ICUWB), 10-12 September 2008, Germany. This work is done under the USCAM-CQ project which is a part of the Ultra Wide Band-enabled Sentient Computing (UWBSC) Research Program funded by Science and Engineering Research Council (SERC), A*STAR, Singapore.

\section{References}

[1] G. N. Shirazi, P.-Y. Kong, and C.-K. Tham, "A cooperative retransmission scheme for IR-UWB networks," in Proceeedings of the IEEE International Conference on Ultra-Wideband (ICUWB '08), vol. 2, pp. 207-210, Hannover, Germany, September 2008.

[2] S. Zhu and K. K. Leung, "Distributed cooperative routing for UWB ad-hoc networks," in Proceedings of IEEE International Conference on Communications (ICC '07), pp. 3339-3344, Glasgow, Scotland, June 2007.

[3] A. Bletsas, H. Shin, and M. Z. Win, "Cooperative communications with outage-optimal opportunistic relaying," IEEE Transactions on Wireless Communications, vol. 6, no. 9, pp. 3450-3460, 2007.

[4] L. Yi and J. Hong, "A new cooperative communication MAC strategy for wireless ad hoc networks," in Proceedings of the 6th IEEE/ACIS International Conference on Computer and Information Science (ICIS '07), pp. 569-574, Melbourne, Australia, July 2007.

[5] A. Azgin, Y. Altunbasak, and G. AlRegib, "Cooperative MAC and routing protocols for wireless ad hoc networks," in Proceedings of IEEE Global Telecommunications Conference (GLOBECOM '05), vol. 5, pp. 2854-2859, St. Louis, Mo, USA, November-December 2005.

[6] IEEE standard part 15.4: Wireless medium access control (MAC) and physical layer (PHY) specifications for low-rate wireless personal area networks (WPANs).

[7] M.-G. Di Benedetto, L. De Nardis, G. Giancola, and D. Domenicali, "The Aloha access (UWB) ${ }^{2}$ protocol revisited for IEEE 802.15.4a," ST Journal of Research, vol. 4, no. 1, pp. 131$142,2007$. 

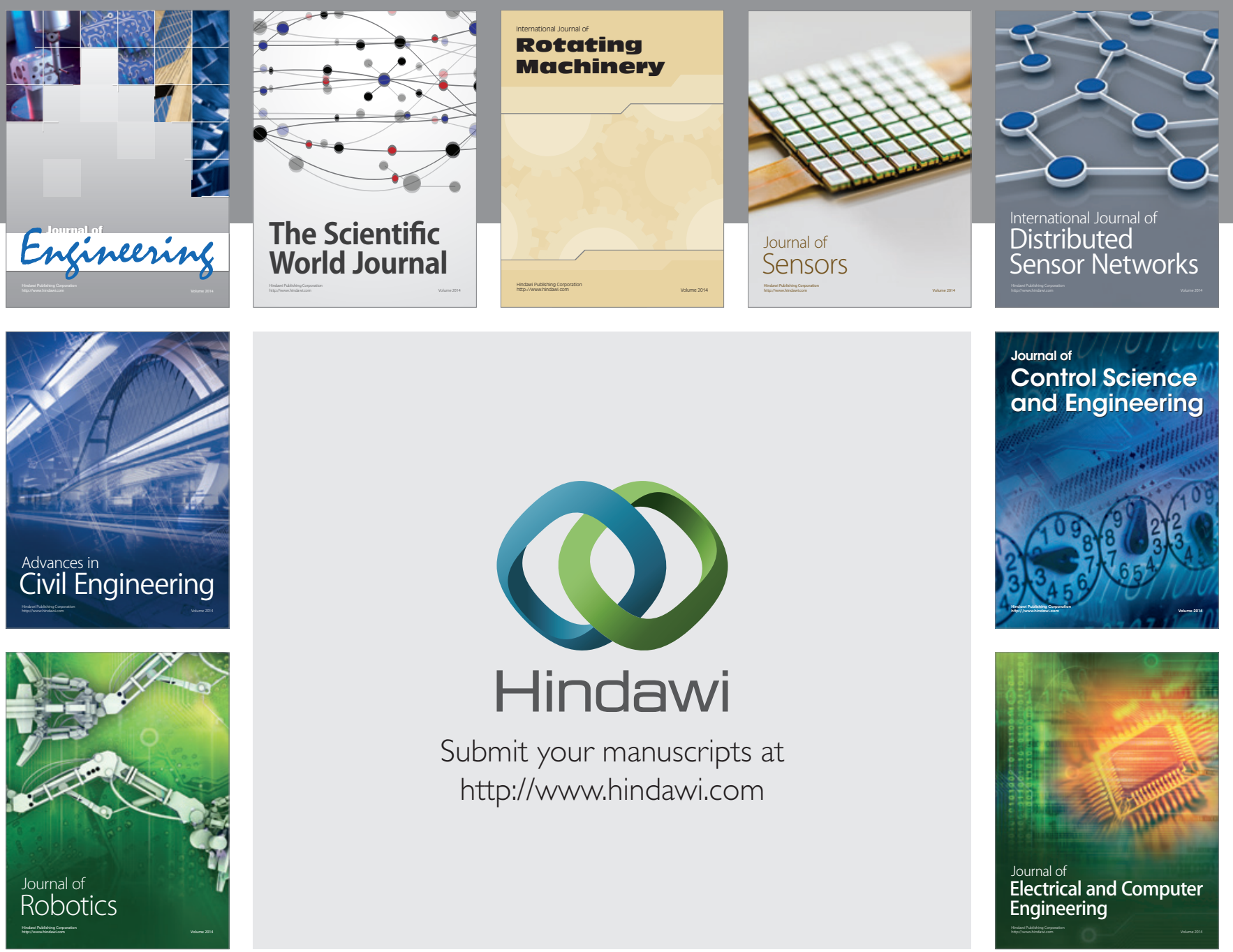

Submit your manuscripts at

http://www.hindawi.com
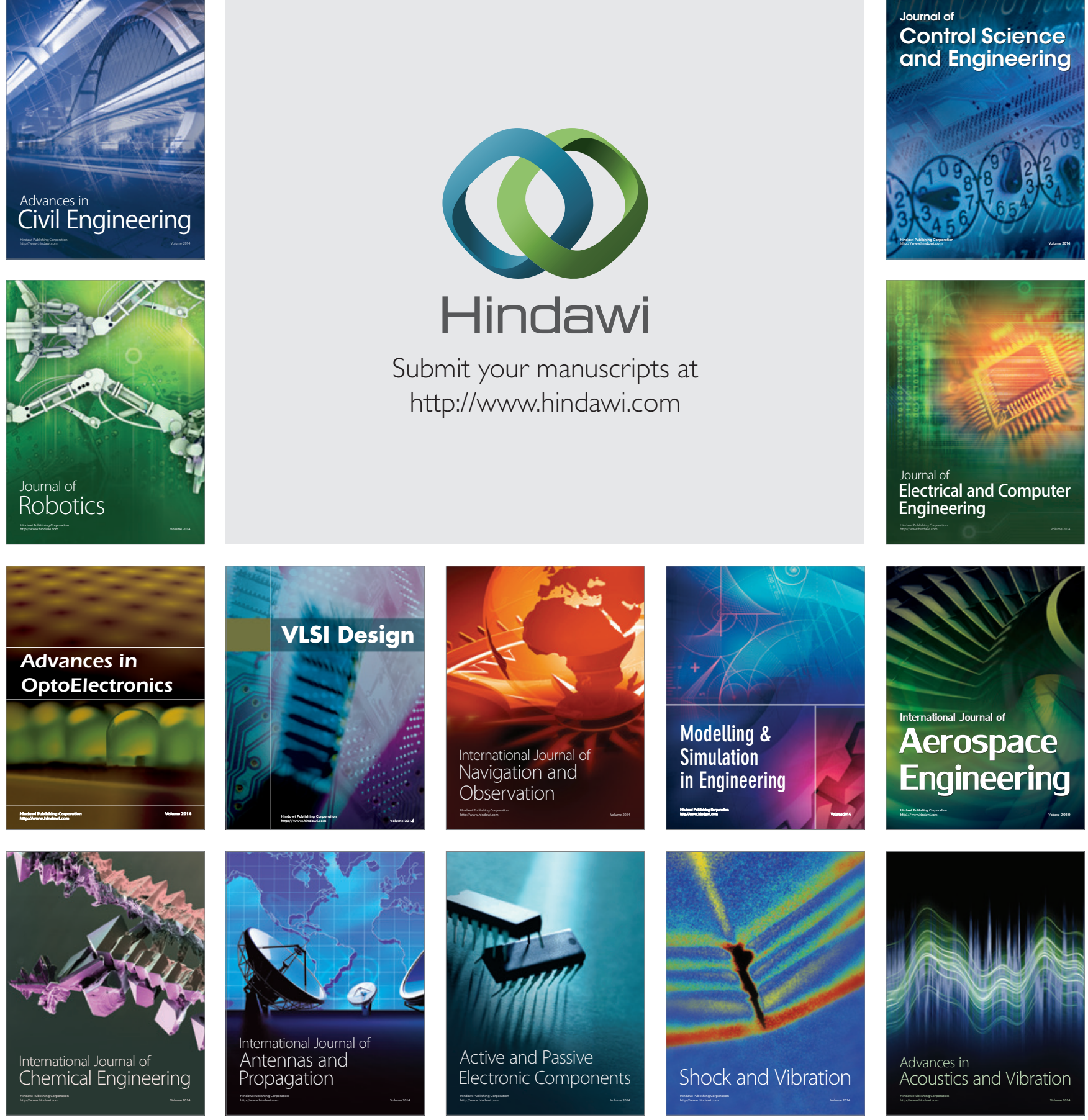